\title{
STOCHASTIC ANALYSIS OF RANDOM AD HOC NETWORKS WITH MAXIMUM ENTROPY DEPLOYMENTS
}

\author{
Thomas Bourgeois $^{1}$ and Shigeru Shimamoto ${ }^{1}$ \\ ${ }^{1}$ Graduate School of Global Information and Telecommunication Studies \\ Waseda University, Japan.
}

\begin{abstract}
In this paper, we present the first stochastic analysis of the link performance of an ad hoc network modelled by a single homogeneous Poisson point process (HPPP). According to the maximum entropy principle, the single HPPP model is mathematically the best model for random deployments with a given node density. However, previous works in the literature only consider a modified model which shows a discrepancy in the interference distribution with the more suitable single HPPP model. The main contributions of this paper are as follows. 1) It presents a new mathematical framework leading to closed form expressions of the probability of success of both one-way transmissions and handshakes for a deployment modelled by a single HPPP. Our approach, based on stochastic geometry, can be extended to complex protocols. 2) From the obtained results, all confirmed by comparison to simulated data, optimal PHY and MAC layer parameters are determined and the relations between them is described in details. 3) The influence of the routing protocol on handshake performance is taken into account in a realistic manner, leading to the confirmation of the intuitive result that the effect of imperfect feedback on the probability of success of a handshake is only negligible for transmissions to the first neighbour node.
\end{abstract}

\section{KEYWORDS}

Ad Hoc Networks · Point Process · Stochastic Geometry .

Medium Access Control

\section{INTRODUCTION}

An ad hoc wireless network consists of self-organizing transceivers communicating with one another in a decentralized way. Such network is referred to as random when the location of nodes in the $d$-dimensional Euclidean space $\mathbb{R}^{d}$ is statistically random [6,21,1]. Using tools from stochastic geometry, the mathematical analysis of carefully chosen models can shed light on the behaviour of a Randomly distributed Ad Hoc Network (RAHN) and can provide insights into the design of Medium Access Control (MAC) and routing protocols [22,23]. The most popular spatial distribution used up to now to model large RAHNs has been the homogeneous Poisson Point Process (HPPP). In a HPPP of intensity $\lambda$, the number of nodes $N(B)$ in a given area $B \subset \mathbb{R}^{d}$ is Poisson distributed with mean $\lambda|B|$, where I.I denotes the $d$-dimensional volume. More point processes and their applications are described in [2], however in this work we focus our analysis on HPPP-based deployments. The popularity of the HPPP is mainly due to its tractability. A less often mentioned fact is that it has maximum entropy among all point processes of a given average rate parameter (i.e, density $\lambda$ ) [8]. Indeed, from the principle of maximum entropy, and with no 
prior knowledge about the distribution of nodes in the network, it is best to choose a model with a minimum of prior information built in $[15,16]$. Consequently,

in an ad hoc network using the "single HPPP" model, hence the motivation for the present work.

The main contributions of this paper are as follows. 1) It presents a new mathematical framework leading to closed form expressions of the probability of success of both one-way transmissions and handshakes for a deployment modelled by a single HPPP. Our approach, based on stochastic geometry, can be extended to complex protocols. 2) From the obtained results, all confirmed by comparison to simulated data, optimal PHY and MAC layer parameters are determined and the relations between them is described in details. 3) The influence of the routing protocol on handshake performance is taken into account in a realistic manner, leading to the confirmation of the intuitive result that the effect of imperfect feedback on the probability of success of a handshake is only negligible for transmissions to the first neighbor node.

Parts of this work were presented in [7]. However, the present paper provides complete, revisited proofs, more detailed explanations as well as additional simulation results. Throughout the paper, we aim to obtain the probability of success of a transmission (one-way and handshake) to the $k$-th nearest neighbour in an ad hoc network modelled by a single HPPP in which nodes employ Slotted-ALOHA (without and with Acknowledgement, respectively). The remainder of this paper is organized as follows. In Section 2, we present our general system model and some preliminary results regarding interference modelling in a network modelled by a single HPPP. In section 3, exploiting results from section 2, we calculate the transmission success probability between two arbitrarily chosen nodes in the network. Reasonable approximations are used to render the analysis tractable. In section 4, we apply the results of the sections 2 and 3 to the cases of the MAC protocols S-ALOHA and S-ALOHA with ACK packet. In section 5, we validate the approximate theoretical expressions obtained in the previous section by comparing them to computer simulation results and analyse the conditions for their validity. Finally, in section 6, a conclusion summarizes the paper.

\section{System Model AND Preliminary Results}

\subsection{Deployment and channel models}

In the remainder, independently of the protocols considered and before specific roles (i.e, transmitter or receiver) are attributed, node deployment is always assumed to be modelled by a ddimensional HPPP with intensity $\lambda$. The channel model incorporates path-loss and fast fading. The path-loss will follow the unbounded power law $l(r)=r^{-\alpha}$ where $\alpha$ is called the path-loss coefficient. The fading is assumed to be Rayleigh distributed, the square thereof then following an exponential distribution with rate parameter equal to one. Transmissions in the network will be slotted and the fading is assumed to be constant over the duration of a slot and independent from one slot to another. Moreover, we always assume fading coefficients of all links to be independent from one another, independent of node position and identically distributed. Since our goal is to analyse the influence of a large number of simultaneous transmissions on the network performances, we will also assume the network to be interference-limited. Hence, as in [12,4], a transmission will be considered successful if the received signal power $\mathrm{S}$ from the intended transmission is greater than the interference I generated by all other transmissions by a factor larger than a given threshold $\theta$. That is, the inequality $\frac{S}{T}>\theta$ must be true for a transmission to be successful. 


\subsection{Interference model}

Let us consider a given network node denoted as $R x$. For any realization of the HPPP, we can sort other nodes by order of distance to $R x$. Let us define for a given slot the random squared fading coefficient $G_{i}, i \in\{1, \ldots,+\infty\}$ of the channel between the $i$-th closest node and $R x, R_{i}$ the random distance from the $i$-th closest node to $R x$. Furthermore, for all realizations of the HPPP, let us assume that the $n$-th closest node has a packet for $R x$ and that all nodes except $R x$ transmit during the slot. For a receiver threshold $\theta$, the probability for this intended transmission to be successful is given by

$$
p_{r x}^{n}(\theta)=\mathbb{E}\left\{\mathbb{P}\left(G_{n}>\theta R_{n}^{\alpha} \sum_{i=1, i \neq n}^{\infty} G_{i} R_{i}^{-\alpha} \mid \vec{R}_{1, \infty}, \vec{G}_{1, \infty \backslash n}\right)\right\}(1)
$$

where $p_{r x}^{n}(\theta)$ denotes the transmission success probability in a "receiver-centric" approach, with the $n$-th neighbour being the transmitter and $\mathbb{E}\{$.$\} is the expectation operator over the random$ vectors $\vec{R}_{1, \infty}=\left[R_{1}, \ldots, R_{\infty}\right]^{T}$ and $\vec{G}_{1, \infty \backslash n}=\left[G_{1}, \ldots, G_{n-1}, G_{n+1}, \ldots, G_{\infty}\right]^{T}$. Then, given that the Complementary Cumulative Distribution Function of an exponential variable with rate parameter one is $F^{c}(x)=e^{-x}$, we obtain from (1),

$$
p_{S}(\theta)=\mathbb{E}_{R_{n}, I}\left\{e^{-\theta R_{n}^{\alpha} I} \mid R_{n}, I\right\}=\mathbb{E}_{R_{n}}\left\{L_{I}\left(\theta R_{n}^{\alpha}\right) \mid R_{n}\right\}
$$

where $I=\sum_{i=1, i \neq n}^{\infty} G_{i} R_{i}{ }^{\alpha}$ is the interference term and $L_{I}(s)=\mathbb{E}_{I}\left\{e^{-s I}\right\}$ is its Laplace Transform. In the following subsections, we determine the Laplace Transform of the interference originating from two important node sets.

\subsection{Interference from the $\mathbf{n}-1$ closest interferers}

Keeping the scenario from the last subsection, consider the $n-1$ closest nodes to $R x$. These nodes are uniformly distributed over a d-dimensional ball with random radius $R_{n}$ deprived from its center. However, given that the volume occupied by the center point (where $R x$ is located) is null, we may as well consider the $\mathrm{n}-1$ nodes to be uniform over the whole ball. Thus, the $\mathrm{n}-1$ nodes form a BPP with random intensity $\lambda=\frac{n-1}{c_{d} R_{n}^{d}}$ where $c_{d}$ indicates the volume of a $d$ dimensional ball with unit radius. In these conditions, we have the following property.

Proposition 1: Consider the annular region $\mathcal{A}(R x, A, B)$ with center $R x$, inner radius $A$ and outer radius $B<R_{n}$. Given that there are exactly $k$ interferers in the annular region $\mathcal{A}(R x, A, B)$, the Laplace Transform of the interference they generate at $R x$ is given by:

$$
L_{I_{\mathcal{A}(R x, A, B)} \mid k}(s)=\left(1+\frac{B^{d} \Omega(s, B)-A^{d} \Omega(s, A)}{B^{d}-A^{d}}\right)^{k}
$$

with

$$
\Omega(s, X)=\frac{F\left(1,1,1-\frac{d}{\alpha}, \frac{s X^{-\alpha}}{1+s X^{-\alpha}}\right)}{1+s X^{-\alpha}}-B\left(1-\frac{d}{\alpha}, 1+\frac{d}{\alpha}\right) s^{\frac{d}{\alpha}} X^{-d}-1(4)
$$


where $F(a, b, c, x)$ is the Gaussian hypergeometric function and $B(a, b)$ denotes the Beta function. To ease numerical computation, a simple approximation of $\Omega(s, X)$ is proposed in section IV.

Proof: See appendix A.

The Laplace Transform of the interference at $R x$ originating from all nodes in the ball, save the center, can be obtained by setting $B=R_{n}, k=n-1$ and making $A$ converge toward zero. Then, to obtain a closed form expression, one may use (3) and the following proposition.

Proposition 2: The limit of $A^{d} \Omega(s, A)$ for $A$ converging toward zero is given by

$$
\lim _{A \rightarrow 0^{+}} A^{d} \Omega(s, A)=0
$$

Proof: See appendix B.

We note also that setting $s=\theta r^{\alpha}, A=a r$ and $B=b r$ where $a, b>0$ in (4) renders the Laplace Transforms in (3) independent of $r$, for any $r>0$. This property will prove useful when considering (2). Hence, for notational convenience, we will define the following function. For any positive real numbers $a$ and $b$ such that $a \leq b, \Phi(\theta, a, b)=L_{I_{\mathcal{A}\{(, a r, b r\rangle} \mid 1}\left(\theta r^{\alpha}\right)$ for all $r>0$. Note that, for the sake of brevity, $\Phi(\theta, a, b)$ will be denoted as $\Phi(a, b)$ in the following when the threshold considered is clear from the context.

\subsection{Interference from nodes beyond the $n$-th neighbour}

Keeping the scenario presented in subsection 2.2, we now consider all nodes further away from $R x$ than the $n$-th closest node. In these conditions, we have the following property.

Proposition 3: The Laplace Transform of the interference generated by all nodes away from $R x$ by a distance greater than $R_{n}$ is given by

$$
L_{I_{\mathbb{R}^{d} / \mathcal{B}\left(R x, R_{n}\right)}}(s)=e^{-\lambda c_{d} R_{n}^{d}\left(\Omega\left(s, R_{n}\right)+B\left(1-\frac{d}{\alpha}, 1+\frac{d}{\alpha}\right) s \frac{d}{\alpha} R_{n}^{-d}\right)}
$$

Proof: See appendix C.

In the following sections, we present general expressions of transmission success probabilities for various scenarios in the single HPPP model.

\section{Transmission Success Probability in an ad hoc network modelled as a single HPPP}

\subsection{Neighbour index definition}

In this subsection, we introduce the notion of neighbour index. As mentioned briefly in introduction, although employing the PGFL leads to a simple expression of the transmission success probability, it is only valid for a given transmitter-receiver distance. However, in the case of a single HPPP, this distance is random with an unknown distribution (since both nodes are part of the original generating HPPP). Conditioning transmitter and receiver on their mutual neighbour indexes allows defining the distance distribution and thus enables averaged results to be inferred. The neighbour index is defined as follows. 
Definition 1: For two distinct network nodes $T x$ and $R x, T x$ has neighbour index $n \in \mathbb{N}^{*}$ with respect to $R x$, which we denote $\mathcal{I}_{R x}(T x)=n$, if there exists exactly $n-1$ nodes closer from $R x$ than $T x$. If the identity of nodes is clear from the context, one may simply write $\mathcal{I}=n$.

From this definition, two different approaches can be considered.

In the receiver-centric approach, transmitters with a packet intended for a given receiver (namely, "intended transmitters", as opposed to "interferers") are conditioned on their neighbour index with respect to the considered receiver node. In the transmitter-centric approach, it is the receiver which is conditioned on its neighbour index with respect to the intended transmitter.

The following conditional probability mass function (p.m.f) $\mathcal{F}(n, k)$ will prove useful in order to relate both approaches. For $T x$ and $R x$ being two distinct network nodes, we define

$$
\mathcal{F}(n, k)=\mathbb{P}\left(\mathcal{I}_{T x}(R x)=n \mid \mathcal{I}_{R x}(T x)=k\right)
$$

When the identity of nodes is clear from the context, we will use simply $\mathcal{T}$. instead of $\mathcal{I}_{T x}(R x)$ and its symmetric $\mathcal{I}_{\text {sym }}$ instead of $\mathcal{I}_{R x}(T x)$ to denote neighbour indexes.

Proposition 4: For points taken from a $d$-dimensional HPPP, the p.m.f in (7)

is given by

$$
\mathcal{F}(n, k)=\sum_{l=0}^{m-1} w_{n, k}(l)(8)
$$

where

$$
w_{n, k}(l)=\left(\begin{array}{c}
k-1 \\
l
\end{array}\right)\left(\begin{array}{c}
n+k-l-2 \\
k-1
\end{array}\right) \frac{\beta_{d}^{l}\left(1-\beta_{d}\right)^{n+k-2 l-2}}{\left(2-\beta_{d}\right)^{n+k-l-1}}
$$

And $m=\min (n, k)$. Also, for $\|T x R x\|=x \in \mathbb{R}^{*+}$ and two $d$-dimensional balls $\mathcal{B}_{d}(T x, x)$ and $\mathcal{B}_{d}(R x, x), \beta_{d}=\beta_{d}$ defined as $\beta_{d}=\frac{|\mathcal{B}(T x, x) \cap \mathcal{B}(R x, x)|}{\left|\mathcal{B}\left(T^{\prime} x, x\right)\right|}=B_{\frac{3}{4}}\left(\frac{d+1}{2}, \frac{1}{2}\right)$ with $B_{x}(a, b)$ denoting the incomplete Beta function.

Proof: The proof for (8), if slightly incorrect, was given in [18]. Indeed,

in this reference, the author did not consider that the number of points in $\mathcal{B}(T x, x) \cap \mathcal{B}(R x, x)$ is at mostmin $(n, k)-1$. Nevertheless, this is a minor error which does not invalidate the thought process of the proof provided, hence the omission of its presentation in this paper. However, we provide in Appendix D the proof that the p.m.f as defined above does satisfy the normalization condition. End of proof.

Also, for notational convenience, we will define for the remainder $[y]_{p}=p y+1-p$, which will prove useful when dealing with the expected value $\mathbb{E}\left\{y^{X}\right\}$ where $y \in \mathbb{R}$ and $X$ is binomially distributed according to $X \sim \mathfrak{B}(N, p)$.

In the following subsections, we show how the preliminary results developed above may be used to obtain the transmission success probability in various contexts. 


\subsection{Receiver-centric approach for a single intended transmission}

In this subsection, we consider the scenario described in subsection 2.2.

Theorem 1: Given that $\mathcal{I}_{R x}(T x)=n$, the probability of success for a transmission from $T x$ to $R x$ is given by

$$
p_{r x}^{n}(\theta)=\frac{\Phi(0,1)^{n-1}}{\left(1+\Omega\left(\theta x^{\alpha}, x\right)+B\left(1-\frac{d}{\alpha}, 1+\frac{d}{\alpha}\right) \theta^{\frac{d}{\alpha}}\right)^{n}}
$$

Proof: The proof relies on the observation that, according to the independence of the number of nodes in disjoint areas in a HPPP, the transmission success probability can be written as follows.

$$
p_{r x}^{n}(\theta)=\mathbb{E}_{R_{n}}\left\{\prod_{j=1}^{\infty} L_{I_{\mathcal{S}_{j}\left(R_{n}\right)}}\left(\theta R_{n}^{\alpha}\right)\right\}
$$

where $R_{n}$ is the distance from $\operatorname{Rxto} T x$ and $\left\{\mathcal{S}_{j}\left(R_{n}\right)\right\}_{j=1, \ldots, \infty}$ is a complete partition of the $d$ dimensional space into disjoint areas functions of $R_{n}$ and $I_{\mathcal{S}_{j}\left(R_{n}\right)}$ is the interference contribution from the nodes located in the area $\mathcal{S}_{j}\left(R_{n}\right)$. The final result is obtained by finding a suitable decomposition of the space.

The $n-1$ "close" interferers located in $\mathcal{B}\left(R x, R_{n}\right)$ form an homogeneous BPP, while the "far" interferers (i.e, away from $R x$ by a distance greater than $R_{n}$ ) form an inhomogeneous PPP . Let us define the following regions: $\mathcal{S}_{n-}=\mathcal{B}\left(R x, R_{n}\right), \mathcal{S}_{n+}=\overline{\mathcal{B}\left(R x, R_{n}\right)}$. These two regions being disjoint, their node distributions are independent, thus the interference $I_{n-}\left(R_{n}\right)$ at $R x$ due to the "close" interferers and the interference $I_{n+}\left(R_{n}\right)$ at $R x$ due to the "far" interferers are independent. As a consequence, from (6) and from (3) for $A \rightarrow 0^{+}, B=R_{n}$ with $n-1$ nodes in $\mathcal{A}(R x, A, B)$, we obtain that the success probability of a transmission from $T x$ to $R x$ can be expressed as

$$
\begin{aligned}
& p_{S}\left(\theta \mid \mathcal{I}_{R x}(T x)=n\right)=\mathbb{E}_{R_{n}}\left\{L_{I_{n-}\left(R_{n}\right)}\left(\theta R_{n}^{\alpha}\right) L_{I_{n+}\left(R_{n}\right)}\left(\theta R_{n}^{\alpha}\right)\right\} \\
& =\mathbb{E}_{R_{n}}\left\{\Phi(0,1)^{n-1} e^{-\lambda c_{d} R_{n}^{d}\left(\Omega\left(\theta R_{n}^{\alpha}, R_{n}\right)+B\left(1-\frac{d}{\alpha}, 1+\frac{d}{\alpha}\right) \theta^{\frac{d}{\alpha}}\right)}\right\}
\end{aligned}
$$

From [18], the distance in a d-dimensional PPP from a node to its $n$-th nearest neighbour is governed by the probability density function (p.d.f )

$$
f_{R_{n}}(r)=d \frac{\left(\lambda c_{d}\right)^{n}}{\Gamma(n)} r^{d n-1} e^{-\lambda c_{d} r^{d}}
$$

Taking the expectation of (11) with respect to $R_{n}=\|T x R x\|$ thus leads to an integral of the form $\int_{0}^{+\infty} r^{d n-1} e^{-\mu r^{d}} d r$ where $\mu>0$, which can be solved easily by applying the variable substitution $y=\mu r^{d}$ and then recognizing in the ensuing integral the Gamma function $\Gamma(n)$. The final result follows then easily, which completes the proof. End of proof.

\subsection{Transmitter-centric approach for a single intended transmission}

We now consider the transmitter-centric approach. 
Theorem 2: The probability of a successful transmission from $T x$ to $R x$, conditioned on $\mathcal{I}_{T x}(R x)=k$, can be approximated by ${ }^{1}$

$$
p_{t x}^{k}(\theta) \approx \frac{\Phi(1,2)^{k-1}}{(1+K(\theta))^{k}} \mathbb{E}_{\mathcal{I}}\left\{\Phi(0,1)^{\mathcal{I}-1}\left[\Phi(1,2)^{-1}\right]_{\beta_{d}}^{\min (\mathcal{I}, k)-1} \mid \mathcal{I}_{\text {sym }}=k\right\}
$$

where $K(\theta)=2^{d} \Omega\left(\theta x^{\alpha}, 2 x\right)+B\left(1-\frac{d}{\alpha}, 1+\frac{d}{\alpha}\right) \theta^{\frac{d}{\alpha}}+\left(2^{d}-2+\beta_{d}\right)(1-\Phi(1,2))$ where $p_{t x}^{k}(\theta)$ denotes the transmission in a "transmitter-centric" approach, with the $k$-th neighbour being the receiver.

Proof: Provided that $\mathcal{I}_{T x}(R x)=k, \mathcal{I}_{R x}(T x)$ is a random variable with distribution given by (7) and $R=\|T x R x\|$ is distributed according to (12) in which $n$ is replaced by $k$. Under these conditions, we consider the following partition of $\mathbb{R}^{d}$.

$$
\begin{aligned}
& \mathcal{S}_{1}(R)=\mathcal{B}_{d}(R x, R) \cap \mathcal{B}_{d}(T x, R) \\
& \mathcal{S}_{2}(R)=\mathcal{B}_{d}(R x, R) \cap \overline{\mathcal{B}_{d}(T x, R)} \\
& \mathcal{S}_{3}(R)=\overline{\mathcal{B}_{d}(R x, R) \cap \mathcal{B}_{d}(T x, R)} \\
& \mathcal{S}_{4}(R)=\mathcal{A}(R x, R, 2 R) \cap \overline{\mathcal{S}_{3}}
\end{aligned}
$$

The geometry of these zones is illustrated in figure 1 . In the following, we simply denote $\mathcal{S}_{j}(R)$ as $\mathcal{S}_{j}$ for the sake of brevity.

We observe that $\mathcal{N}\left(\mathcal{S}_{1}\right)$ is a binomially distributed random variable $\mathfrak{B}\left(\mathcal{M}-1, \beta_{d}\right)$, where $\mathcal{M}=\min \left(\mathcal{I}_{R x}(T x), k\right)$. Partitioning the space using the above-defined regions, the transmission success probability can be expressed as

$$
\begin{aligned}
& p_{l x}^{k}(\theta)=\mathbb{E}_{R, \mathcal{I}_{R x}(T x)}\left\{\Phi(0,1)^{\mathcal{I}_{R x}(T x)-1} \mathbb{E}_{\mathcal{N}\left(\mathcal{S}_{1}\right)}\left\{L_{I_{\mathcal{S}_{3}}}\left(\theta R^{\alpha}\right)\right\}\right. \\
& \left.\mathbb{E}_{\mathcal{N}\left(\mathcal{S}_{4}\right)}\left\{L_{I_{\mathcal{S}_{4}}}\left(\theta R^{\alpha}\right)\right\} e^{-\lambda c_{d} R^{d}\left(2^{d} \Omega\left(\theta R^{\alpha}, 2 R\right)+B\left(1-\frac{d}{\alpha}, 1+\frac{d}{\alpha}\right) \theta^{\frac{d}{\alpha}}\right)}\right\}
\end{aligned}
$$

We note that $L_{I_{\mathcal{S}_{3}}}\left(\theta R^{\alpha}\right)$ is dependent on $\mathcal{N}\left(\mathcal{S}_{1}\right)$, through the relation $\mathcal{N}\left(\mathcal{S}_{3}\right)=k-1-\mathcal{N}\left(\mathcal{S}_{1}\right)$ while the remaining functions are only dependent on $\mathcal{I}_{R x}(T x)$ and $R$. From the properties of HPPPs, the number of nodes in $\mathcal{S}_{4}$ is Poisson distributed and independent of the number of nodes in other regions.

The distance from $R x$ to any node uniformly distributed in $\mathcal{S}_{3}$ (or in $\mathcal{S}_{4}$, respectively) follows a distribution with a non-trivial expression, rendering difficult any attempt to compute a closed form expression for the Laplace Transform of the interference contribution from nodes located in these regions. So as to keep the calculations tractable, the distribution of the distance from $R x$ to a node in the above-mentioned regions is approximated by the distribution of the distance from $R x$ to a node uniformly deployed over the annular region $\mathcal{A}(R x, R, 2 R)$.

A reasonable justification for this approximation is as follows. In order to obtain a simple approximation regarding a distribution, one may successively "forget" part of the constraints on the actual distribution and select the maximum-entropy distribution fitting the remaining

\footnotetext{
${ }^{1}$ The expectation in the expression of transmission success probability for the transmitter-centric approach is on the discrete random variable $\mathcal{I}$, which leads to an infinite sum. In practice, computing the first few terms produces values very close to the actual result.
} 
constraints, if it exists. In our case, removing information regarding the shapes of $\mathcal{S}_{3}$ and $\mathcal{S}_{4}$, the only remaining knowledge we have is that both zones are inside $\mathcal{A}(R x, R, 2 R)$. However, the uniform distribution is the maximum entropy distribution among all continuous distributions supported in a bounded set, given that no other statistical characteristics are known about the actual distribution. Consequently, it is also the optimal distribution estimate for the actual distribution of nodes, according to the maximum entropy principle.

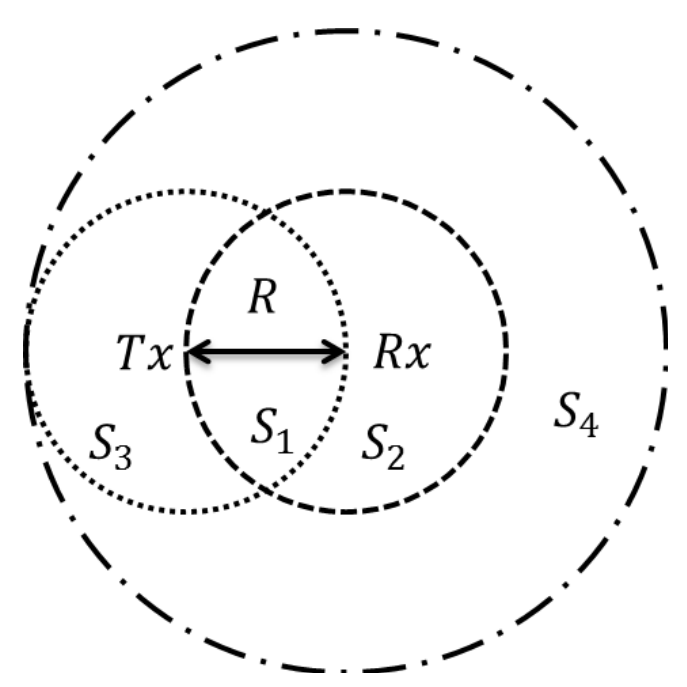

Figure 1 Illustration of the interference situation at a receiver $R x$ given an intended transmitter $T x$ located at distance $R$. The different zones in the figure are defined in (14).

As a result, the interference generated by a given number of nodes in $\mathcal{S}_{3}$ (or $\mathcal{S}_{4}$ ) may be approximated by the interference due to the same number of nodes uniformly distributed in $\mathcal{A}(R x, R, 2 R)$.

Using this approximation for $\mathcal{S}_{3}$, we obtain

$$
\begin{aligned}
& \mathbb{E}_{\mathcal{N}\left(\mathcal{S}_{1}\right)}\left\{L_{I_{\mathcal{S}_{3}}}\left(\theta R^{\alpha}\right)\right\} \approx \sum_{i=0}^{\mathcal{M}-1}\left(\begin{array}{c}
\mathcal{M}-1 \\
i
\end{array}\right) \beta_{d}^{i}\left(1-\beta_{d}\right)^{\mathcal{M}-1-i} \Phi(1,2)^{k-1-i} \\
& =\Phi(1,2)^{k-1}\left(\frac{\beta_{d}}{\Phi(1,2)}+1-\beta_{d}\right)^{\mathcal{M}-1}
\end{aligned}
$$

where the last line follows from the binomial theorem.

Regarding $\mathcal{S}_{4}$, given that $\left|\mathcal{S}_{4}\right|=\left(2^{d}-2+\beta_{d}\right) c_{d} R^{d}$, we have from the definition of the Poisson distribution that

$$
\begin{aligned}
& \mathbb{E}_{\mathcal{N}\left(\mathcal{S}_{4}\right)}\left\{L_{I_{\mathcal{S}_{4}}}\left(\theta x^{\alpha}\right)\right\} \approx e^{-\lambda\left(2^{d}-2+\beta_{d}\right) c_{d} R^{d}} \sum_{j=0}^{+\infty} \frac{\left(\lambda\left(2^{d}-2+\beta_{d}\right) c_{d} R^{d}\right)^{j}}{j !} \Phi(1,2)^{j} \\
& =e^{-\lambda c_{d} R^{d}\left(2^{d}-2+\beta_{d}\right)(1-\Phi(1,2))}
\end{aligned}
$$


Further taking the expectation over $\mathcal{I}_{R x}(T x)$ and then taking the expectation over $R$ in (15) leads to an integral that can be solved in the exact same way as the one leading to (9), which completes the proof. End of proof.

In the next section, we show how results calculated up to now may be applied to the analysis of performances of some simple Medium Access Control protocols for ad hoc networks.

\section{APPLiCATiOn to the ANAlysis OF SlotTED-ALOHA}

In this section, we analyse the success probability of transmissions in an ad hoc network employing the MAC protocols Slotted-ALOHA with and without ACK packet. The system model used in the following is as described in subsection 2.1.

\subsection{Case of S-ALOHA with constant Medium Access Probability}

In previous sections, we considered that all nodes in the network were active transmitters, except for a single receiver $R x$. However, node activity in real networks is controlled by the MAC protocol.

In a Slotted-ALOHA (S-ALOHA) protocol with fixed Medium Access Probability (MAP) $p$, each node in the network with a packet to transmit does so during the next slot if it passes a random test with success probability $p$, and stays silent otherwise [14]. We assume in the following that all silent nodes can act as receiver. Let us further assume that nodes always have at least one packet ready for transmission and that consequently all nodes passing the random test do transmit. Then, let us consider an ad hoc network modelled as an HPPP $\mathcal{X}$ with constant intensity $\lambda$. In this case, employing an S-ALOHA protocol with constant MAP $p$ results, from the thinning properties of PPPs [12], in the separation of the original HPPP into two distinct HPPPs. Namely, $\mathcal{X}_{1}$ with intensity $p \lambda$ and $\mathcal{X}_{0}$ with intensity $(1-p) \lambda$, which correspond to active transmitters and silent nodes, respectively.

The expressions of transmission success probability obtained in the previous section can be adapted easily to the case of Slotted-ALOHA with constant MAP by taking into account the following two observations. 1) The independent thinning of the original process $\mathcal{X}$ has for consequence that, for any given bounded set $\mathcal{S} \subset \mathbb{R}^{d}$, the number of active transmitters $\mathcal{N}_{1}(\mathcal{S})$ in $\mathcal{S}$ is binomially distributed (i.e $\mathcal{N}_{1}(\mathcal{S}) \sim \mathfrak{B}(\mathcal{N}(\mathcal{S}), p)$ ). 2) For unbounded regions of $\mathbb{R}^{d}$, active transmitters located in them form an inhomogeneous PPP with intensity $p \lambda$. It is easily verified that the Laplace Transform of their interference contribution may be obtained from the case treated in the previous section merely by changing $\lambda$ into $p \lambda$ in the equations.

In the next subsection, we analyse the case of Slotted-ALOHA with Acknowledgement.

\subsection{Case of constant MAP S-ALOHA with ACK slots}

We now consider the same system as in the previous subsection, except that now each data transmission time slot is followed by a slot reserved for the transmission of ACK packets. Thus, nodes which receive successfully a packet intended for them during a data slot send an ACK packet during the following ACK slot.

Although interference in a HPPP is spatially correlated, we assume in the following that transmission successes on different links are independent so as to keep the derivations of handshake performances tractable. So as to show this assumption is reasonable, simulated data used for comparison will take into account the spatial correlation of interference. 
An acknowledgement packet is considered successful if its SIR ratio is greater than the threshold $\theta^{\prime}$. If an intended data packet and the following ACK packet are both correctly received, then the handshake is considered successful. We assume that fading coefficients in data slots and ACK slots are independent.

Also, we define $q(\theta)$ the spatially averaged probability for any node in the network not transmitting during a given data slot to send a packet during the following ACK slot. That is, $q(\theta)$ can be seen as the spatially averaged probability that a node receives successfully a packet intended for itself during a given data slot, conditioned on its belonging to the receiver set. As a consequence, $q(\theta)$ not only depends on the MAP $p$, but also on the routing protocol employed (i.e, the way nodes in the network choose their packet destinations). For a given receiver $R x \in \mathcal{X}_{0}$, the general expression for $q(\theta)$ is given by

$$
q(\theta)=p \sum_{n=1}^{\infty} \mathbb{P}\left(X_{n} \rightarrow R x\right) p_{r x}^{n}(\theta)
$$

in which $X_{n}$ denotes the $n$-th nearest node to $R x, X_{n} \rightarrow R x$ means " $X_{n}$ selects $R x$ as destination" and where $p_{r x}^{n}(\theta)$ is the transmission success probability from $X_{n}$ to $R x$.

Consequently, provided that the routing protocol employed allows $\mathbb{P}\left(X_{n} \rightarrow R x\right)$ to be known for all $n$, it is possible to determine $q(\theta)$ using the above equation. The particular case in which transmitters choose their destination uniformly among their $N$ nearest neighbours will be covered in the next section.

For a given probability $q(\theta)$, which we denote simply as $q$, we have the following theorem.

Theorem 3: Conditioned on $\mathcal{I}_{T x}(R x)=k$, the probability for a data packet from a transmitter $T x$ to a receiver $R x$ and the following ACK packet from $R x$ to $T x$ to be both successfully received can be approximated by

$$
\begin{aligned}
& p_{l x}^{k}\left(\theta, \theta^{\prime} \mid \mathcal{I}_{T x}(R x)=k\right) \approx \Psi_{2}\left(\theta, \theta^{\prime}, 1\right)^{k-1} \\
& \frac{\mathbb{E}_{\mathcal{I}}\left\{\Psi_{1}\left(\theta, \theta^{\prime}, 1\right)^{\mathcal{I}-1}\left[\frac{\Psi_{3}\left(\theta, \theta^{\prime}, 1\right)}{\Psi_{1}\left(\theta, \theta^{\prime}, 1\right) \Psi_{2}\left(\theta, \theta^{\prime}, 1\right)}\right]_{\beta_{d}(1)}^{\min (\mathcal{I}, k)-1}\right\}}{\left(1+\sum_{i=2}^{\infty} \Upsilon\left(\theta, \theta^{\prime}, i\right)\right)^{k}}
\end{aligned}
$$

Where

$$
\begin{aligned}
& \Psi_{1}\left(\theta, \theta^{\prime}, i\right)=\left[\Phi\left(\theta^{\prime}, i, i+1\right)\right]_{q}\left[\frac{\Phi(\theta, i-1, i)}{\left[\Phi\left(\theta^{\prime}, i, i+1\right)\right]_{q}}\right]_{p} \\
& \Psi_{2}\left(\theta, \theta^{\prime}, i\right)=\left[\Phi\left(\theta^{\prime}, i-1, i\right)\right]_{q}\left[\frac{\Phi(\theta, i, i+1)}{\left[\Phi\left(\theta^{\prime}, i-1, i\right)\right]_{q}}\right]_{p} \\
& \Psi_{3}\left(\theta, \theta^{\prime}, i\right)=\left[\Phi\left(\theta^{\prime}, i-1, i\right)\right]_{q}\left[\frac{\Phi(\theta, i-1, i)}{\left[\Phi\left(\theta^{\prime}, i-1, i\right)\right]_{q}}\right]_{p} \\
& \Upsilon\left(\theta, \theta^{\prime}, i\right)=\left(i^{d}-\right)\left(2-\Psi_{1}\left(\theta, \theta^{\prime}, i\right)-\Psi_{2}\left(\theta, \theta^{\prime}, i\right)\right) \\
& +\left(\beta_{d}(i)-2(i-1)^{d}+\beta_{d}(i-1)\right)\left(1-\Psi_{3}\left(\theta, \theta^{\prime}, i\right)\right)
\end{aligned}
$$

and where $\beta_{d}(i)=\frac{|\mathcal{B}(T x, i x) \cap \mathcal{B}(R x, i x)|}{\left|\mathcal{B}\left(T^{\prime} x, i x\right)\right|}=B_{\frac{1}{i}-\frac{1}{4 i^{2}}}\left(\frac{d+1}{2}, \frac{1}{2}\right)$. 


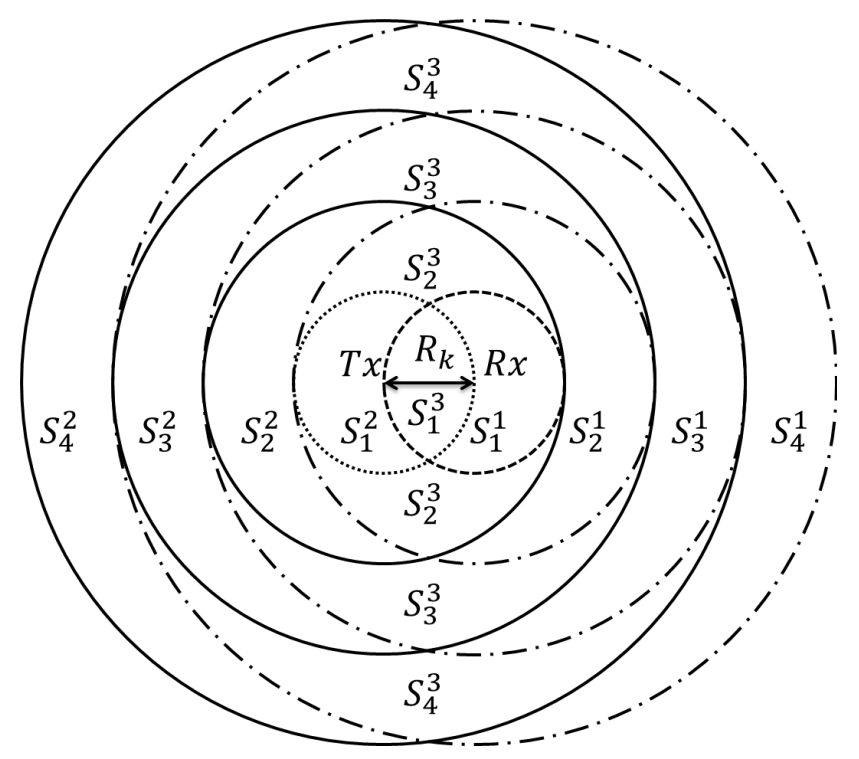

Figure 2 : Illustration of the space partition used for the analysis of transmission success probability using Slotted-ALOHA protocol with Acknowledgement packet. The partition is composed of the disjoint regions resulting from the intersections of two infinite sets of concentric circles, centred on $T x$ and $R x$, respectively. The figure only shows the regions closest to $T x$ and $R x$. Regions further away are defined in the same way.

Proof: Given that $\mathcal{I}_{T x}(R x)=k$, let us consider the partition $\left\{\mathcal{S}_{i}^{j}(R)\right\}_{j=1,2,3 ; i=1, \ldots, \infty}$ of $\mathbb{R}^{d}$, which is illustrated in figure 2 . In this case the joint probability of success of a data transmission and its following ACK transmission is given by

$$
p_{l x}^{k}\left(\theta, \theta^{\prime}\right)=\mathbb{E}\left\{\prod_{j_{1}, i_{1}} L_{I_{i_{1}}^{j_{1}, \text { Data }}}\left(\theta R^{\alpha}\right) \prod_{j_{2}, i_{2}} L_{S_{S_{i_{2}}, A c k}^{j_{2}}}\left(\theta R^{\alpha}\right)\right\}
$$

where the expectation is over $R, \mathcal{I}_{R x}(T x),\left\{\mathcal{N}_{1}\left(\mathcal{S}_{i}^{j}\right)\right\}_{j=1,2,3 ; i=1, . ., \infty}$ and $\left\{\mathcal{N}\left(\mathcal{S}_{i}^{j}\right)\right\}_{j=1,2,3 ; i=1, \ldots, \infty}$. The first infinite product is actually the conditional probability of success of the data transmission, while the second product is that of the ACK transmission.

In [20], the authors employ product inequalities to separate the two transmit directions and derive lower and upper bounds. However, in our model, it is possible to treat directly the dependency between them. We can rewrite the handshake success probability using a single product as

$$
\begin{aligned}
& p_{t x}^{n}\left(\theta, \theta^{\prime}\right)= \\
& \mathbb{E}\left\{\prod_{j, i} L_{I_{S_{i}^{j}, \text { Data }}}\left(\theta R^{\alpha}\right) L_{I_{\mathcal{S}_{i}^{j}, A c k}}\left(\theta R^{\alpha}\right)\right\}
\end{aligned}
$$

In order to keep the analysis tractable, we will use for each of the regions in the partition the same approximation on the distance distribution as we used to obtain (14). Note that according to the definition of $q$, the number of ACK packet transmitters in a given region $\mathcal{S}$ is binomially distribution according to $\mathfrak{B}\left(\mathcal{N}(\mathcal{S})-\mathcal{N}_{1}(\mathcal{S}), q\right)$.

Consequently, we have 


$$
\begin{aligned}
& L_{I_{\mathcal{S}_{i}^{1}, \text { Data }}}\left(\theta R^{\alpha}\right) \approx \Phi(\theta, i-1, i)^{\mathcal{N}_{1}\left(\mathcal{S}_{i}^{1}\right)} \\
& L_{I_{\mathcal{S}_{i}^{2}, \text { Data }}}\left(\theta R^{\alpha}\right) \approx \Phi(\theta, i, i+1)^{\mathcal{N}_{1}\left(\mathcal{S}_{i}^{2}\right)} \\
& L_{I_{\mathcal{S}_{i}^{3}, \text { Data }}}\left(\theta R^{\alpha}\right) \approx \Phi(\theta, i-1, i)^{\mathcal{N}_{1}\left(\mathcal{S}_{i}^{3}\right)} \\
& L_{I_{\mathcal{S}_{i}^{1}, A c k}}\left(\theta R^{\alpha}\right) \approx[\Phi(\theta, i, i+1)]_{q}^{\mathcal{N}\left(\mathcal{S}_{i}^{1}\right)-\mathcal{N}_{1}\left(\mathcal{S}_{i}^{1}\right)} \\
& L_{I_{\mathcal{S}_{i}^{2}, \text { Ack }}}\left(\theta R^{\alpha}\right) \approx[\Phi(\theta, i-1, i)]_{q}^{\mathcal{N}\left(\mathcal{S}_{i}^{2}\right)-\mathcal{N}_{1}\left(\mathcal{S}_{i}^{2}\right)} \\
& L_{I_{\mathcal{S}_{i}^{3}, A c k}}\left(\theta R^{\alpha}\right) \approx[\Phi(\theta, i-1, i)]_{q}^{\mathcal{N}\left(\mathcal{S}_{i}^{3}\right)-\mathcal{N}_{1}\left(\mathcal{S}_{i}^{3}\right)}
\end{aligned}
$$

Also, $\mathcal{N}_{1}(\mathcal{S})$ is binomially distributed according to $\mathfrak{B}(\mathcal{N}(\mathcal{S}), p)$. Thus, replacing the above terms into (22) and taking the expectation over $\left\{\mathcal{N}_{1}\left(\mathcal{S}_{i}^{j}\right)\right\}_{j=1,2,3 ; i=1, \ldots, \infty}$, we obtain

$$
p_{\text {tx }}^{k}\left(\theta, \theta^{\prime}\right) \approx \mathbb{E}\left\{\prod_{i=1}^{\infty} \prod_{j=1}^{3} \Psi_{j}\left(\theta, \theta^{\prime}, i\right)^{\mathcal{N}\left(\mathcal{S}_{i}^{j}\right)}\right\}
$$

Regarding the terms $\mathcal{N}\left(\mathcal{S}_{i}^{j}\right)$, we observe that $\mathcal{N}\left(\mathcal{S}_{1}^{3}\right)$ is binomially distributed according to $\mathfrak{B}\left(\min \left(\mathcal{I}_{R x}(T x), k\right)-1, \beta_{d}(1)\right) \quad$. Also, $\quad \mathcal{N}\left(\mathcal{S}_{1}^{2}\right)=\mathcal{I}_{R x}(T x)-1-\mathcal{N}\left(\mathcal{S}_{1}^{1}\right) \quad$ and $\mathcal{N}\left(\mathcal{S}_{1}^{3}\right)=k-1-\mathcal{N}\left(\mathcal{S}_{1}^{1}\right)$. Denoting $\mathcal{I}=\mathcal{I}_{R x}(T x)$, we then have

$$
\begin{aligned}
& \mathbb{E}_{\mathcal{I}, \mathcal{N}\left(\mathcal{S}_{1}^{1}\right)}\left\{\prod_{j=1}^{3} \Psi_{j}\left(\theta, \theta^{\prime}, 1\right)^{\mathcal{N}\left(\mathcal{S}_{1}^{j}\right)}\right\}=\Psi_{2}\left(\theta, \theta^{\prime}, 1\right)^{k-1} \\
& \mathbb{E}_{\mathcal{I}}\left\{\Psi_{1}\left(\theta, \theta^{\prime}, 1\right)^{\mathcal{I}-1}\left[\frac{\Psi_{3}\left(\theta, \theta^{\prime}, 1\right)}{\Psi_{2}\left(\theta, \theta^{\prime}, 1\right) \Psi_{2}\left(\theta, \theta^{\prime}, 1\right)}\right]_{\beta_{d}(1)}^{\min (\mathcal{I}, k)-1}\right\}
\end{aligned}
$$

The other terms $\left\{\mathcal{N}\left(\mathcal{S}_{i}^{j}\right)\right\}_{j=1,2,3 ; i>1}$ are independent of $\mathcal{I}_{R x}(T x)$, independent of one another and Poisson distributed with parameter $\lambda\left|\mathcal{S}_{i}^{j}\right|$.

Consequently, for $i>1$ and any $j \in\{1,2,3\}$,

$$
\mathbb{E}_{\mathcal{N}\left(\mathcal{S}_{i}^{j}\right)}\left\{\Psi_{j}\left(\theta, \theta^{\prime}, i\right)\right\}=e^{-\lambda\left|S_{i}^{j}\right|\left(1-\Psi_{j}\left(\theta, \theta^{\prime}, i\right)\right)}
$$

Also, the area of the regions $\left|S_{i}^{j}\right|$ are given by

$$
\begin{aligned}
& \left|S_{i}^{1}\right|=\left|S_{i}^{2}\right|=\left(i^{d}-\beta_{d}(i)\right) c_{d} R^{d} \\
& \left|S_{i}^{3}\right|=\left(\beta_{d}(i)-2(i-1)^{d}+\beta_{d}(i-1)\right) c_{d} R^{d}
\end{aligned}
$$

Consequently, using the above and then taking the expectation with respect to $R$, we obtain an integral of the same type as the one leading to (14). Solving this integral leads directly to the final result, which completes the proof.

Given that most of the important results calculated until now are actually approximations, it is reasonable to compare the obtained formulae with the results of computer simulations. 


\section{Simulation Results}

\subsection{Simulation Method}

The method we use in our computer program to simulate an infinite HPPP is that proposed in [12]. In each simulation drop, we generate a random deployment then record the success/failure of a given link. Results are finally averaged over all the deployment realizations.

The performance metric used is the Mean Maximum Achievable Spectral Efficiency (MMASE) $\eta_{t x}^{k}$ conditioned on $\mathcal{I}_{T x}(R x)=k$, given by

$$
\eta_{t x}^{k}\left(\theta, \theta^{\prime}\right)=p(1-p) \log _{2}(1+\theta) p_{t x}^{k}\left(\theta, \theta^{\prime}\right)
$$

where the dependence on $\theta^{\prime}$ disappears in the case no acknowledgement packet is used. In order to ease the computation of theoretical expressions, we use the following approximation for the function $\Omega(s, X)$.

$$
\tilde{\Omega}(s, X)=\left(\left(1+s X^{-\alpha}\right)^{\frac{d}{\alpha}}-\left(s X^{-\alpha}\right)^{\frac{d}{\alpha}}\right) B\left(1-\frac{d}{\alpha}, 1+\frac{d}{\alpha}\right)
$$

The above approximation is obtained easily from the limit property of the Hypergeometric function used in Appendix C.

\subsection{Simulation results}

In the following, we compare the theoretical results obtained in the previous sections with results from computer simulations. All theoretical results on figures are represented by solid lines, while dots are the simulated data. The path-loss exponent is $\alpha=4, d=2$ and $\lambda=10^{-3}$ nodes per square-meter. On some figures (i.e when there was a noticeable difference), we plotted both theoretical results using the above approximation (solid lines) and using the expression with Hypergeometric function given in (4) (dashed lines).

Case of S-ALOHA without ACK: In figure 3 and figure 4, we consider the case of S-ALOHA in a uniform RAHN modelled as a 2-dimensional HPPP. We observe a good agreement between the behaviours of simulated and approximate theoretical results. The latter appear as upper bounds of the simulated ones, which is to be expected since the approximation used in the derivation of (20) overestimates the distance between some interferers and the receiver.

Figure 3 illustrates the trade-off between decodability and bit rate. Note that the optimal MCS for transmissions to a given neighbour depends on the operating MAP p. Conversely, for a given receiver threshold, intuition suggests the existence of an optimal MAP. This is confirmed in figure 4. Note that for $p>0.5$, the number of receiver nodes is on average smaller than the number of transmitters, which implies that the optimal $\mathrm{p}$ lies between 0 and 0.5 . We note that both theoretical and simulated results reach their respective maximum almost at the same abscissa, in both figures. Regarding the influence of $\theta$ on optimal MAP $p$, further investigations have showed that increasing the data packet receiver threshold $\theta$ decreases the optimal $p$, which follows directly from the trade-off between the density of transmissions and the individual transmission rate.

Case of S-ALOHA with ACK: Regarding S-ALOHA with acknowledgement, we assume a routing protocol which makes transmitters select their packet destination uniformly among their $N$ closest neighbours. In this particular case, $q(\theta)$ is given as follows. 


$$
q(\theta)=\frac{p}{N} \sum_{k=1}^{N} p_{t x}^{k}(\theta)
$$

where $p_{t x}^{k}(\theta)$ denotes the success probability for a transmission to the $k$-th nearest neighbour and is given by (19) where $\theta^{\prime}=0$. We assume $N=2$ in the following. The performances of SALOHA with ACK packet are described in figure 5. The theoretical results are computed using (19) and (29). The denominator in (19) is approximated by computing the first thirty terms of the infinite sum (more terms did not provide any additional noticeable accuracy gain).

We observe again a good agreement between the behaviors of simulated and approximate theoretical results. The latter do not explicitly upper or lower bound simulated results, which can be explained by the diversity of shape of the regions on which the approximation for the distance from interferers to the receiver is used. The existence of optimal operating points can be noted, in a similar fashion to S-ALOHA. Again, both theoretical and simulated results reach their respective maximum at abscissa very close from each other.

Regarding the influence of $\theta^{\prime}$ on optimal MAP $p$ and $\theta$, although it is not shown here due to the lack of space, increasing $\theta^{\prime}$ also increases the optimal MAP $p$. This can be justified in the same way as the influence of $\theta$ on $p$ in the case of S-ALOHA without acknowledgement, by considering that the number of interferers during the ACK packet slot is proportional to $1-p$.

Also, by comparing figure 5 and figure 6 , we observe that increasing $\theta^{\prime}$ actually increases the value of the optimal $\theta$, although the achieved maximum MMASE is lower than with a smaller $\theta^{\prime}$. One possible justification for this result is that a higher $\theta$ leads to less interference during the ACK slot, thus allowing a higher $\theta^{\prime}$ to be used.

Finally, comparing Fig 3, Fig 5 and Fig 6, we observe that the transmission of the ACK packet has negligible influence for the case $k=1$, even for high values of $\theta^{\prime}$. Note however, that for $k>1$, the influence of imperfect feedback becomes stronger (e.g for $k=3, \theta^{\prime}=0 \mathrm{~dB}$, the MMASE in the case of S-ALOHA with ACK is $75 \%$ of the case without ACK). Our observations confirm the intuitive fact that imperfect feedback is only negligible for transmissions between close neighbors.

\section{Conclusion}

In this paper, we analyzed the probability of success of a transmission (one-way and handshake) to the $k$-th nearest neighbor in an ad hoc network modelled by a single homogeneous Poisson Point Process in which nodes employ the Slotted-ALOHA MAC protocol (without and with acknowledgement, respectively). From the principle of maximum entropy, the single HPPP is the best deployment model for ad hoc networks with a constant node density. Previous works so far have been limited to a simplified deployment model, for tractability reasons. However, in this work, we presented a tractable mathematical analysis enabling the analysis of spatially averaged network performances at the routing level while tackling directly the dependency between both transmit directions in handshakes. We compared the developed close-form formula with computer simulation results and concluded that this work can find applications in the joint quantitative study of some MAC and routing protocols. Notably, exploiting the notion of neighbor index, we confirmed through our results the intuitive fact that imperfect feedback in handshakes is only negligible for transmissions to/from the closest neighbor. 


\section{Appendix A}

In this appendix, we provide the proof of (3). From [13] (eq.(4)), we have

$$
L_{I}(s)=\left(\frac{d}{B^{d}-A^{d}} \int_{A}^{B} x^{d-1} L_{G}\left(s x^{-\alpha}\right) d x\right)^{k}
$$

The Laplace transform of the squared fading is given by

$$
L_{G}(s)=\frac{1}{1+s}(32)
$$

Replacing the above in (31), leads to the integral given by

$$
J=\int_{A}^{B} \frac{x^{d-1} d x}{1+s x^{-\alpha}}=\int_{A}^{B} u(x) d x=\int_{0}^{B} u(x) d x-\int_{0}^{A} u(x) d x
$$

Note that through the variable substitution $t=1-\left(\frac{x}{y}\right)^{\alpha}$, each of the integrals in the last expression of (34) can also be expressed as

$$
\int_{0}^{y} u(x) d x=\frac{y^{d} \int_{0}^{1}(1-t)^{\frac{d}{\alpha}}\left(1-\frac{1}{1+s y^{-\alpha}} t\right)^{-1} d t}{\alpha\left(1+s y^{-\alpha}\right)}
$$

where $y$ takes the value $A$ or $B$. One can recognize in (34) Euler's integral

transform for the Gauss hypergeometric function[17], given by (35).

$$
F(a, b, c, z)=\frac{\int_{0}^{1} t^{b-1}(1-t)^{c-b-1}(1-z t)^{-a} d t}{B(b, c-b)}
$$

where $B(a, b)$ is the Beta function and $F(a, b ; c ; z)$ denotes the Gauss hypergeometric function. It then follows that

$$
\int_{0}^{y} u(x) d x=\frac{y^{d} F\left(1,1,2+\frac{d}{\alpha}, \frac{1}{1+s y^{-\alpha}}\right)}{(\alpha+d)\left(1+s y^{-\alpha}\right)}
$$

Then, one can use the following identity for $z=\frac{s x^{-\alpha}}{1+s x^{-\alpha}}$ to relate the above result to the function $\Omega(s, X)$ as defined earlier in the paper

$$
\frac{F\left(1,1,2+\frac{d}{\alpha}, 1-z\right)(1-z) \frac{d}{\alpha}}{1+\frac{d}{\alpha}}=F\left(1,1,1-\frac{d}{\alpha}, z\right)(1-z)-B\left(1-\frac{d}{\alpha}, 1+\frac{d}{\alpha}\right)\left(\frac{z}{1-z}\right)^{\frac{d}{\alpha}}
$$

Using (37) and (36) in (31) leads to the final result, which completes the proof.

\section{Appendix B}

In this appendix we provide the proof of (5). We first notice from the properties of the Gauss Hypergeometric function that for $c-a-b<0$, we have [17] 
International Journal of Wireless \& Mobile Networks (IJWMN) Vol. 6, No. 3, June 2014

$$
\lim _{z \rightarrow 1^{-}} \frac{F(a, b, c, z)}{(1-z)^{c-a-b}}=\frac{\Gamma(c) \Gamma(a+b-c)}{\Gamma(a) \Gamma(b)}
$$

Then, using (4), let us rephrase $A^{d} \Omega(s, A)$ as

$$
A^{d} \Omega(s, A)=A^{d} f(s, A)\left(1-\frac{s}{s+1 A^{\alpha}}\right)^{-\frac{d}{\alpha}}-A^{d}-B\left(1-\frac{d}{\alpha}, 1+\frac{d}{\alpha}\right) s^{\frac{d}{\alpha}}
$$

Where $f(s, A)=F\left(1,1,1-\frac{d}{\alpha}, \frac{s}{1 A^{\alpha}+s}\right)\left(1-\frac{s}{s+1 A^{\alpha}}\right)^{1+\frac{d}{\alpha}}$. We observe that by setting $z=\frac{s}{s+1 \Lambda^{\alpha}}$ and $a=1, b=1, c=1-\frac{d}{\alpha}$, we obtain easily the limit of $f(s, A)$ for $A$ converging toward zero from (38). From there, the final result easily follows, which completes the proof.

\section{Appendix C}

In this appendix we provide the proof of (6). From [12] (section 3.7.1), we have

$$
L_{I_{\mathbb{R} d / \mathcal{B}(O, x)}}(s)=e^{-\lambda c_{d} \mathbb{E}_{G}\left\{s^{\frac{d}{\alpha}} G^{\frac{d}{\alpha}} \gamma\left(1-\frac{d}{\alpha}, s G x^{-\alpha}\right)-x^{d}\left(1-e^{-s G x^{-\alpha}}\right)\right\}}
$$

where $\gamma(n, x)$ denotes the lower incomplete gamma function. Let us rephrase part of the above expression as follows.

$$
\mathbb{E}_{G}\left\{G^{\frac{d}{\alpha}} \gamma\left(1-\frac{d}{\alpha}, s G x^{-\alpha}\right)\right\}=\int_{0}^{+\infty} \frac{1}{\Gamma(1)} t^{\frac{d}{\alpha}} \gamma\left(1-\frac{d}{\alpha}, t s x^{-\alpha}\right) e^{-1 t} d t
$$

The above integral can be solved by replacing initially the incomplete lower gamma function by its equivalent involving the Kummer's confluent hypergeometric function.[17] That is,

$$
\gamma\left(1-\frac{d}{\alpha}, t s x^{-\alpha}\right)=\frac{e^{-t s x^{-\alpha}} M\left(1,2-\frac{d}{\alpha}, t s x^{-\alpha}\right)}{\left(1-\frac{d}{\alpha}\right)\left(t s x^{-\alpha}\right)^{\frac{d}{\alpha}-1}}
$$

Then, replacing (42) into (41) reveals an integral form of the Gauss Hypergeometric function given by

$$
F(a, b, c, z)=\frac{1}{\Gamma(b)} \int_{0}^{+\infty} e^{-t} t^{b-1} M(a, c, t z) d t
$$

which is valid only for $b>0$. Therefore, it follows that

$$
\begin{aligned}
& \mathbb{E}_{G}\left\{G^{\frac{d}{\alpha}} \gamma\left(1-\frac{d}{\alpha}, s G x^{-\alpha}\right)\right\}=\frac{\left(s x^{-\alpha}\right)^{1-\frac{d}{\alpha}} F\left(1,2,2-\frac{d}{\alpha}, \frac{s x^{-\alpha}}{1+s x^{-\alpha}}\right)}{\left(1-\frac{d}{\alpha}\right)\left(1+s x^{-\alpha}\right)^{2}} \\
& =\frac{z s^{-\frac{d}{\alpha}} x^{d}}{\left(1-\frac{d}{\alpha}\right)} F\left(1,2,2-\frac{d}{\alpha}, z\right)(1-z)
\end{aligned}
$$

Where $z=\frac{s x^{-\alpha}}{1+s x^{-\alpha}}$. Note that using the same notation, we have

$$
\mathbb{E}_{G}\left\{1-e^{-s G x^{-\alpha}}\right\}=1-(1-z)
$$

Using the recurrence relations of the Hypergeometric function, one can obtain the following identity

$$
F(1, b, c, t)=\frac{c-1}{b-1}\left(\frac{F(1, b-1, c-1, t)}{t}-\frac{1}{t}\right)
$$


which, by setting $b=2, c=2-\frac{d}{\alpha}$ and $t=z=\frac{s x^{-\alpha}}{1+s x^{-\alpha}}$ lead us to

$$
\mathbb{E}_{G}\left\{G^{\frac{d}{\alpha}} \gamma\left(1-\frac{d}{\alpha}, s G x^{-\alpha}\right)\right\}=s^{-\frac{d}{\alpha}} x^{d}\left(F\left(1,1,1-\frac{d}{\alpha}, z\right)-1\right)(1-z)
$$

Replacing (47) and (45) into (40) we obtain

$$
L_{I_{\mathbb{R} d / \mathcal{B}(O, x)}}(s)=\exp \left(-\lambda c_{d} x^{d}\left(F\left(1,1,1-\frac{d}{\alpha}, z\right)(1-z)-1\right)\right)
$$

which can be expressed as a function of $\Omega(s, x)$ as defined in (4) and thus completes the proof.

\section{Appendix D}

In this appendix, we prove that the p.m.f in (8) satisfies the normalization condition. That is, we aim to prove that

$$
S_{k}=\sum_{n=1}^{\infty} \mathcal{F}(n, k)=1
$$

To do so, we start by reordering the double sum above into

$$
S_{k}=\sum_{l=0}^{k-1} \sum_{n=l+1}^{\infty}\left(\begin{array}{c}
k-1 \\
l
\end{array}\right)\left(\begin{array}{c}
n+k-l-2 \\
k-1
\end{array}\right) \frac{\beta_{d}^{l}\left(1-\beta_{d}\right)^{n+k-2 l-2}}{\left(2-\beta_{d}\right)^{n+k-l-1}}(50)
$$

which is obtained simply by interverting sum indexes after making the term $\min (n, k)$ disappear. Then, sorting terms and using the change of index $n=n^{\prime}+l+1$, we obtain

$$
S_{k}=\frac{1}{1-\beta_{d}}\left(\frac{1-\beta_{d}}{2-\beta_{d}}\right)^{k} \sum_{l=0}^{k-1}\left(\begin{array}{c}
k-1 \\
l
\end{array}\right)\left(\frac{\beta_{d}}{1-\beta_{d}}\right)^{l} \sum_{n=0}^{\infty}\left(\begin{array}{c}
n+k-1 \\
n
\end{array}\right)\left(\frac{1-\beta_{d}}{2-\beta_{d}}\right)^{n}
$$

Using the binomial theorem and recognizing the series expansion of the function $(1-x)^{-k}$ in the second sum, we can transform the above into

$$
S_{k}=\frac{1}{1-\beta_{d}}\left(\frac{1-\beta_{d}}{2-\beta_{d}}\right)^{k} \frac{1}{\left(1-\beta_{d}\right)^{k-1}}\left(2-\beta_{d}\right)^{k}=1
$$

which completes the proof, for all $k \geq 1$. 
International Journal of Wireless \& Mobile Networks (IJWMN) Vol. 6, No. 3, June 2014

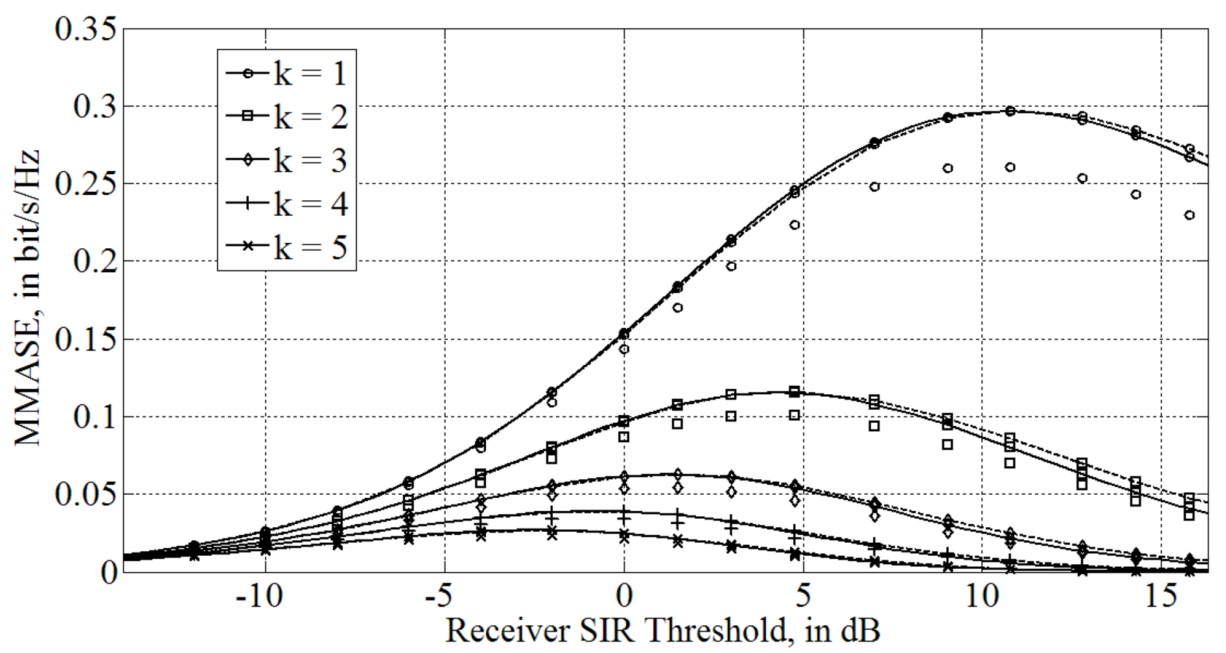

Figure 3 : MMASE of transmissions to the $k$-th neighbour in a 2-dimensional uniform RAHN employing S-ALOHA with fixed MAP $p=0.3$, against the receiver threshold (in $\mathrm{dB}$ ).

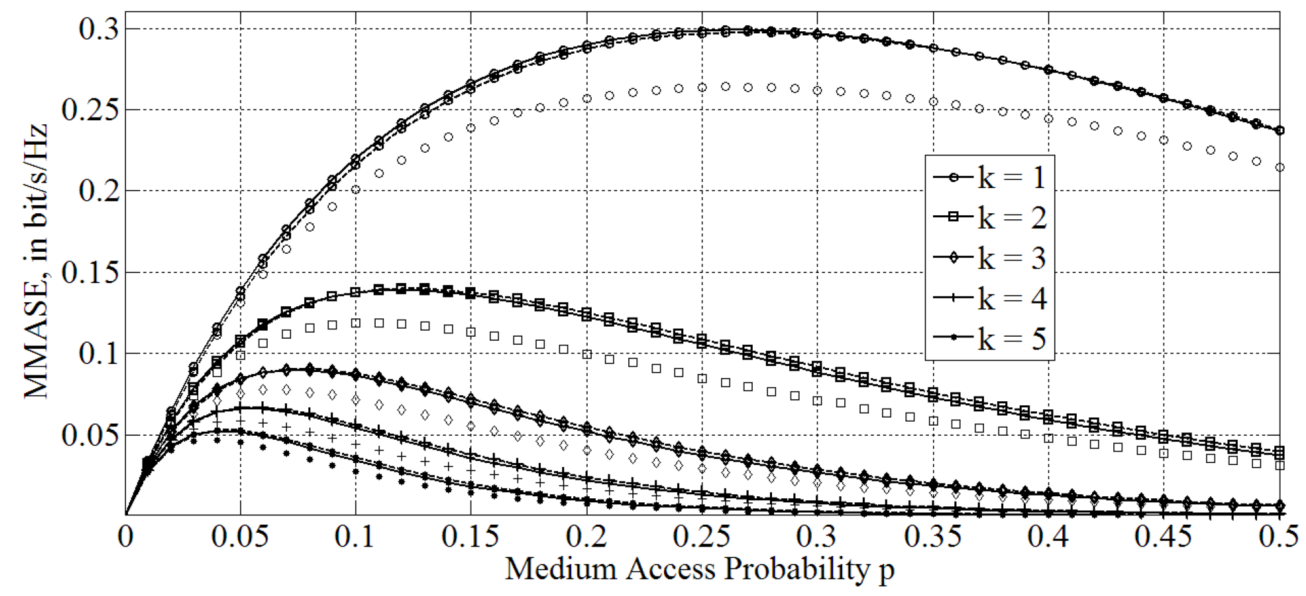

Figure 4 : MMASE of transmissions to the $k$-th neighbour in a 2-dimensional uniform RAHN employing $\mathrm{S}-\mathrm{ALOHA}$, against the MAP $p$, for a given a receiver threshold $\theta=10.4$.

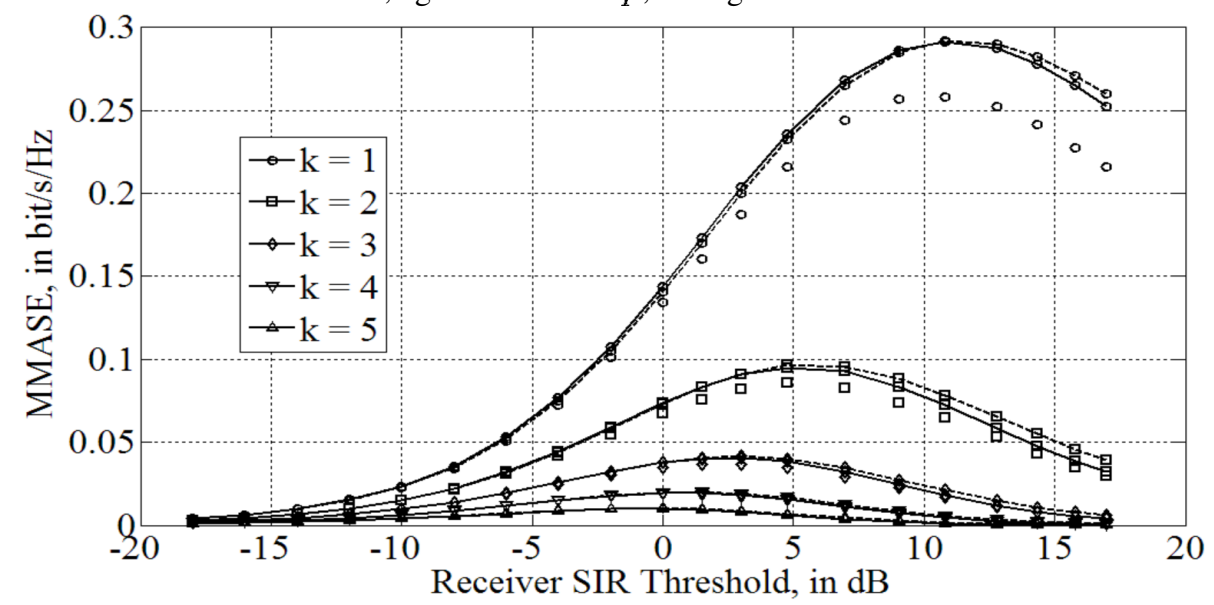

Figure 5 : MMASE of transmissions to the $k$-th neighbour in a 2-dimensional uniform RAHN employing S-ALOHA with ACK and fixed MAP $p=0.3$, against the receiver threshold $\theta$ (in $\mathrm{dB}$ ). The curves are obtained from (19), for $\theta^{\prime}=0 d B$. 
International Journal of Wireless \& Mobile Networks (IJWMN) Vol. 6, No. 3, June 2014

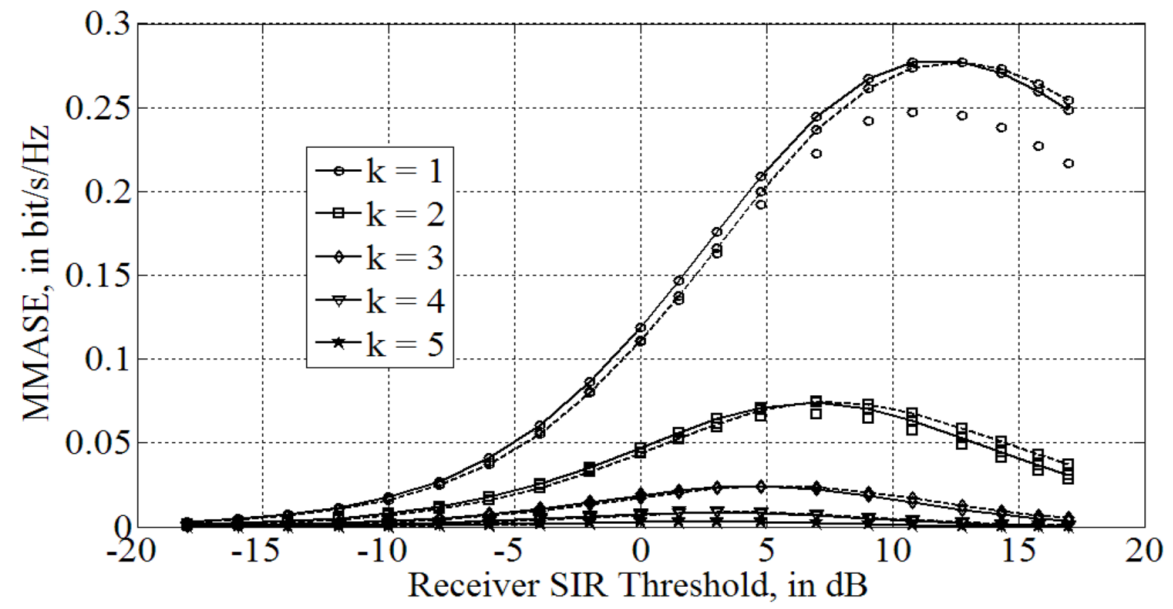

Figure 6 : MMASE of transmissions to the $k$-th neighbour in a 2-dimensional uniform RAHN employing S-ALOHA with ACK and fixed MAP $p=0.3$, against the receiver threshold $\theta$ (in $\mathrm{dB}$ ). The curves are

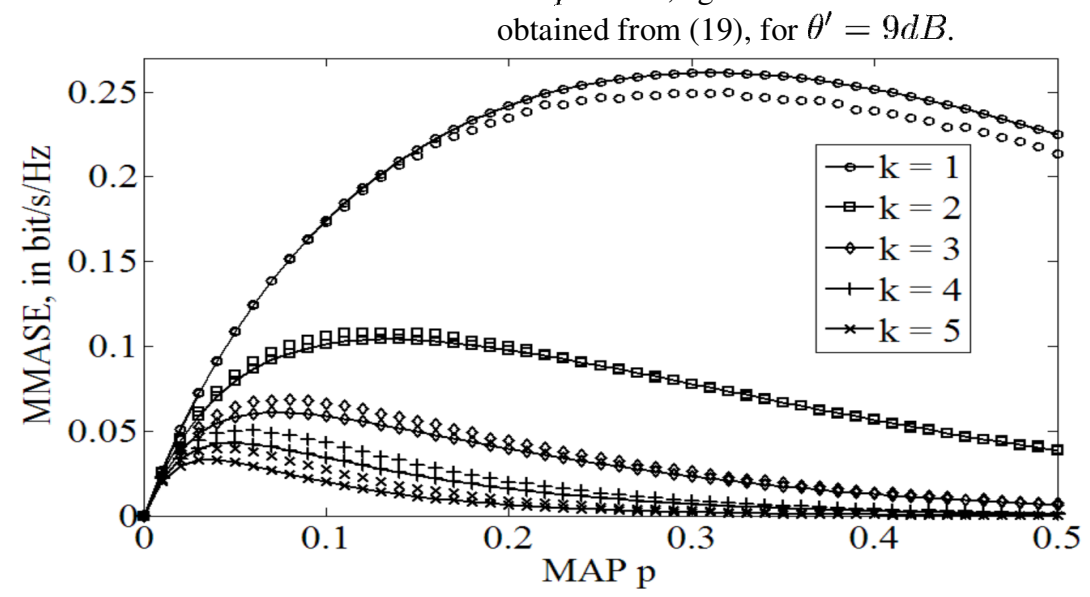

Figure 7 : MMASE of transmissions to the $k$-th neighbour in a 2-dimensional uniform RAHN employing S-ALOHA with ACK packet, against the MAP $p$. The data packet threshold is $\theta=8 d B$ and the ACK packet threshold is $\theta^{\prime}=0 d B$. The curves are obtained from (19).

\section{REFERENCES}

[1] J. Akhtman and L. Hanzo. Heterogeneous networking: An enabling paradigm for ubiquitous wireless communications [point of view]. Proceedings of the IEEE, 98(2):135-138, Feb.

[2] .G. Andrews, R.K. Ganti, M. Haenggi, N. Jindal, and S. Weber. A primer on spatial modeling and analysis in wireless networks. Communications Magazine, IEEE, 48(11):156 -163, november 2010.

[3] Weng Chon Ao and Kwang-Cheng Chen. Bounds and exact mean node degree and node isolation probability in interference-limited wireless ad hoc networks with general fading. Vehicular Technology, IEEE Transactions on, 61(5):2342-2348, 2012.

[4] F. Baccelli, B. Blaszczyszyn, and P. Muhlethaler. An aloha protocol for multihop mobile wireless networks. Information Theory, IEEE Transactions on, 52(2):421 - 436, feb. 2006.

[5] F. Baccelli, Bartlomiej Blaszczyszyn, and P. Muhlethaler. Stochastic analysis of spatial and opportunistic aloha. Selected Areas in Communications, IEEE Journal on, 27(7):1105-1119, 2009.

[6] S. Basagni, M. Conti, S. Giordano, and I. Stojmenovic. Mobile Ad Hoc Networking. Wiley-IEEE Press, 2004.

[7] T. Bourgeois and S. Shimamoto. Stochastic analysis of handshake-type mechanisms in uniformly random wireless ad hoc networks. submitted to Global Telecommunications Conference, 2013. GLOBECOM '13. IEEE.

[8] D.J. Daley and D. Vere-Jones. An Introduction to the Theory of Point Processes. Springer Series in Statistics, 1988. 
International Journal of Wireless \& Mobile Networks (IJWMN) Vol. 6, No. 3, June 2014

[9] R.K. Ganti, J.G. Andrews, and M. Haenggi. High-sir transmission capacity of wireless networks with general fading and node distribution. Information Theory, IEEE Transactions on, 57(5):3100-3116, 2011.

[10] M. Haenggi. A geometric interpretation of fading in wireless networks:Theory and applications. Information Theory, IEEE Transactions on, 54(12):5500-5510, 2008.

[11] M. Haenggi. Outage, local throughput, and capacity of random wireless networks. Wireless Communications, IEEE Transactions on, 8(8):4350-4359, 2009.

[12] M. Haenggi and R. K. Ganti. Interference in large wireless networks. Foundations and Trends in Networking, (2):127 -248, 2009.

[13] M. Haenggi and S. Srinivasa. Modeling interference in finite uniformly random networks. Proc. International Workshop on Information Theory for Sensor Networks (WITS'07), 2007.

[14] H. Inaltekin, Mung Chiang, H.V. Poor, and S.B. Wicker. On unbounded path-loss models: effects of singularity on wireless network performance. Selected Areas in Communications, IEEE Journal on, 27(7):1078-1092, September.

[15] E. T. Jaynes. Information theory and statistical mechanics. Physical Review, 106:620 - 630, May 1957.

[16] E. T. Jaynes. Information theory and statistical mechanics. Physical Review, 108:171 - 191, Oct. 1957.

[17] F. W. J. Olver, D. W. Lozier, R. F. Boisvert, and C. W. Clark, editors. NIST Handbook of Mathematical Functions. Cambridge University Press, New York, NY, 2010.

[18] S.P. Smith. Threshold validity for mutual neighborhood clustering. Pattern Analysis and Machine Intelligence, IEEE Transactions on, 15(1):89 -92, jan 1993.

[19] R. Vaze and R.W. Heath. Transmission capacity of ad-hoc networks with multiple antennas using transmit stream adaptation and interference cancellation. Information Theory, IEEE Transactions on, 58(2):780-792, 2012.

[20] R. Vaze, K.T. Truong, S. Weber, and R.W. Heath. Two-way transmission capacity of wireless ad-hoc networks. Wireless Communications, IEEE Transactions on, 10(6):1966-1975, 2011.

[21] J. Zheng and A. Jamalipour. Wireless Sensor Networks:A Networking Perspective. Wiley-IEEE Press, 2009.

[22] E. Chukwuka and K. Arshad. Energy Efficient MAC Protocols for Wireless Sensor Network: A Survey. International Journal of Wireless and Mobile Networks (IJWMN), 5(4), August 2013

[23] V. Hnatyshin. Improving MANET Routing Protocols Through the Use of Geographical Information. A Survey. International Journal of Wireless and Mobile Networks (IJWMN), 5(2), April 2013

\section{Authors}

Thomas Bourgeois was born in Paris, France, in 1987. He received the Master of Engineering from the French "grande ecole" TELECOM Bretagne, Brest, France, isn 2011. $\mathrm{He}$ is currently working toward the Ph.D. degree with the Graduate School of Global Information and Telecommunications Studies, Waseda University, Tokyo, Japan. His research interests include ad hoc networks, heterogeneous cellular networks, stochastic geometry and information theory.

Shigeru Shimamoto was born in Mie, Japan, in 1963. He received the B.E. and M.E. degrees in communications engineering from the University of Electro-Communications, Tokyo, Japan, in 1985 and 1987, respectively. In April 1987, he joined NEC Corporation, Otsu, Japan. From April 1991 to September 1992, he was an Assistant Professor with the University of Electro-Communications. In 1991, he was an Assistant Professor with the Department of Computer Science, Faculty of Engineering, Gunma University, Maebashi,

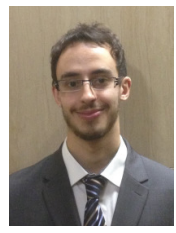
Japan. In 2000, he was an Assistant Professor with the Graduate School of Global Information and Telecommunications Studies, Waseda University, Tokyo, where he has been a Professor since April 2002. In 2008, he was a Visiting Professor with Stanford University, Stanford, CA. His research interests include satellite, mobile communications, and next-generation intelligent transportation systems. Dr. Shimamoto is a member of the Institute of Electrical, Information, and Communication Engineers. 